\title{
A new association between polymorphisms of the SLC6A7 gene in the chromosome 5q31-32 region and asthma
}

\author{
Jeong-Hyun Kim ${ }^{1,6}$, Hyun Sub Cheong ${ }^{2,6}$, Byung-Lae Park², Joon Seol Bae ${ }^{1}$, Seok Jung ${ }^{3}$, Sang-Hyuk Yoon ${ }^{3}$, \\ Jong Sook Park ${ }^{3,4}$, An Soo Jang ${ }^{3,4}$, Sung Woo Park ${ }^{3,4}$, Soo-Taek Uh ${ }^{3}$, Yong-Hoon Kim ${ }^{4}$, Hyeon-Kyu Hwang ${ }^{5}$, \\ Choon-Sik Park ${ }^{3,4}$ and Hyoung Doo Shin ${ }^{1,2}$
}

The human chromosomal 5q31-33 region has been implicated as a susceptibility locus for several immune-mediated diseases including asthma in several populations. Recently, the extraneuronal GABAergic system has been implicated as a new link to airway obstruction in asthma. In addition, the SLC6A7 gene, which is positioned at 5q31-32 and encodes the transporter for an excitatory neurotransmitter of L-proline, has never been studied for its association with asthma. In this study, resequencing of all exon, promoter region ( $2 \mathrm{~kb}$ ), and exon-intron boundary regions in the SLC6A7 gene found a total of 33 single nucleotide polymorphisms (SNPs) in 24 Korean asthmatic patients. After the initial SNP survey, a total of 17 common SNPs with minor allele frequency (MAF) over 10\% were genotyped in 498 asthmatic patients and 303 normal controls. Logistic analyses revealed significant associations between genetic variants of the $S L C 6 A 7$ gene and asthma ( $P$-value up to $6.0 \times 10^{-4}$; $P^{c o r r}$ value up to $0.009)$. In further regression analyses, minor alleles of intronic $+11431 T>C,+12213 C>T$ and $+12927 A>G$ in linkage disequilibrium block 2 and $+20113 T>C$ in $3^{\prime}$ UTR significantly increased the bronchodilator response in asthmatics $(P$-value of recessive model up to 0.008 ; which are not significant after multiple correction). Therefore, our findings suggest that SLC6A7 could be a susceptible gene for asthma.

Journal of Human Genetics (2010) 55, 358-365; doi:10.1038/jhg.2010.34; published online 30 April 2010

Keywords: asthma; bronchodilator response; linkage disequilibrium; single nucleotide polymorphism; SLC6A7

\section{INTRODUCTION}

According to the World Health Organization, it is estimated that over 300 million people worldwide suffer from asthma. As a chronic inflammatory airway disease, asthma is characterized by the infiltration and activation of eosinophils in the lung. ${ }^{1}$ Asthma and its phenotypes are complex traits resulting from interactions between multiple disease susceptibility factors and the surrounding environment, which has a major influence on the onset and severity of asthma. ${ }^{2}$ Despite our advanced knowledge of the pathological basis of asthma, its comprehensive etiology is not fully understood.

Recently, genome-wide association studies have identified susceptible genes for asthma, such as genetic variations of metallopeptidase domain 33 in patients with asthma and bronchial hyper-responsive- ness, phosphodiesterase $4 D$ in childhood asthma, and variants regulating the expression of orosomucoid 1-like 3 in childhood asthma. ${ }^{3-5}$ In addition, the human chromosomal region $5 \mathrm{q} 31-33$, which contains a cluster of cytokines and other immune-related genes, has been mapped as a susceptibility locus for several inflammatory or autoimmune diseases including asthma in a number of populations. ${ }^{6-9}$ Functional variations of genes in chromosomal region $5 \mathrm{q} 31-33$, such as interleukin $(I L)-13, I L-9, I L-12 B$ and $D C N P 1$ were also found to be associated with asthma. ${ }^{10-13}$ However, the exact genetic and functional mechanisms underlying asthmatic pathology remain unclear.

The SLC6A7 gene, which is located at the chromosomal 5q31-33 region, encodes a neurotransmitter L-proline transporter and is a member of the $\gamma$-aminobutyric acid (GABA) neurotransmitter gene

\footnotetext{
${ }^{1}$ Department of Life Science, Sogang University, Seoul, Republic of Korea; ${ }^{2}$ Department of Genetic Epidemiology, SNP Genetics Inc., Seoul, Republic of Korea; ${ }^{3}$ Genome Research Center for Allergy and Respiratory Diseases, Soonchunhyang University Bucheon Hospital, Gyeonggi-do, Republic of Korea; ${ }^{4}$ Division of Allergy and Respiratory Medicine, Soonchunhyang University Bucheon Hospital, Gyeonggi-do, Republic of Korea and ${ }^{5}$ Division of Allergy and Respiratory Disease, Soonchunhyang University Gumi Hospital, Gyeongsangbuk-do, Republic of Korea

${ }^{6}$ These authors contributed equally to this work.

Correspondence: Dr HD Shin, Department of Life Science, Sogang University, Seoul, 121-742, Republic of Korea.

E-mail: hdshin@sogang.ac.kr or Dr C-S Park, Genome Research Center for Allergy and Respiratory Diseases, Division of Allergy and Respiratory Medicine, Soonchunhyang University Bucheon Hospital, Gyeonggi-do, 420-767, Republic of Korea.

E-mail: mdcspark@unitel.co.kr
}

Received 12 January 2010; revised 17 March 2010; accepted 24 March 2010; published online 30 April 2010 
family. As the SLC6A7 gene was first discovered, ${ }^{14,15}$ there have been few reports about its exact functions or molecular mechanisms, except for its expression in the brain regions related to the glutamatergic pathways. ${ }^{16}$ In addition, there has been no evidence of association between SLC6A7 and asthma. However, airway remodeling for the pathogenesis of asthma and its correlation with excitatory neurotransmitters have been suggested. ${ }^{17,18}$ Moreover, new evidence has recently emerged suggesting a link between the extraneuronal signaling system (the excitatory rather than inhibitory GABAergic system) and mucus overproduction in asthma. ${ }^{19,20}$

To investigate this new link between genetic variation(s) of the SLC6A7 gene in the chromosomal 5q31-33 region and asthma, we discovered the polymorphisms of the SLC6A7 gene and studied its association with asthma and related phenotypes.

\section{MATERIALS AND METHODS}

Study subjects

A total of 498 asthmatic patients were recruited from the Asthma Genome Research Center, which consists of three tertiary hospitals in Korea (Soonchunhyang University, Seoul; Bucheon and Chunan Hospitals). Ethical approvals were obtained from the institutional review board of each hospital. All subjects were Korean. All patients had the clinical symptoms and the physical examination results compatible with asthma, based on the Global Initiative for Asthma guidelines. ${ }^{21}$ All patients showed a history of dyspnea and wheezing during the previous 12 months, plus one of the following: (1) $>15 \%$ increase in $\mathrm{FEV}_{1}$ or $>12 \%$ increase plus $200 \mathrm{ml}$ following inhalation of a shortacting bronchodilator, (2) $<10 \mathrm{mg} \mathrm{ml}^{-1}$ PC20 methacholine, or (3) $>20 \%$ increase in $\mathrm{FEV}_{1}$ following 2 weeks of treatment with inhaled steroids and longacting bronchodilators. Airway reversibility was applied to rapid improvements in $\mathrm{FEV}_{1}$, measured at $15 \mathrm{~min}$ after inhalation of a rapid-acting bronchodilator (400 mg salbutamol (albuterol), MDI).22 Bronchodilator-induced change of $\mathrm{FEV}_{1}$ was measured by the difference of post $\mathrm{FEV}_{1}(\mathrm{l} / \mathrm{sec})$ minus pre $\mathrm{FEV}_{1}$ $(1 / \mathrm{sec})$ divided by pre-bronchodilator $\mathrm{FEV}_{1}(1 / \mathrm{sec})$ and represented as percentages ((post $\mathrm{FEV}_{1}$ minus pre $\left.\mathrm{FEV}_{1}\right) /$ pre-bronchodilator $\left.\mathrm{FEFV}_{1} \times 100\right)$. We evaluate the association between the bonchodilator-induced change and genotypes using regression analysis. Acute or chronic eosinophilic pneumonia and Churg-Strauss syndrome were excluded from the study population. A total of 303 normal subjects were recruited from the general population and spouses of the patients who answered negatively to a screening questionnaire for respiratory symptoms and had $\mathrm{FEV}_{1}$ greater than $75 \%$ predicted, the provoca- tion concentration causing a fall in the $\mathrm{FEV}_{1}$ of $20 \%$ (PC20) by methacholine greater than $10 \mathrm{mg} \mathrm{ml}^{-1}$, and normal findings on a simple chest radiogram.

Total immunoglobulin E (IgE) and specific IgE to Dermatophagoides farinae and D. pteronyssinus were measured using the UniCAP system (Pharmacia Diagnostics, Uppsala, Sweden). Twenty-four common inhalant allergens (for example, dust mites (Dermatophagoides farinae and D. pteronyssinus), cat fur, dog fur, cockroaches, grasses, trees and ragweed pollen) (Bencard Co. Ltd., Brentford, UK) were used for a skin prick test. Atopy was defined as having a wheal reaction by allergen extract that was equal to or greater than that by histamine $\left(1 \mathrm{mg} \mathrm{ml}^{-1}\right)$, or $3 \mathrm{~mm}$ in diameter. The clinical parameters are summarized in Table 1.

\section{Resequencing and genotyping}

Genomic DNA was isolated from the peripheral blood of patients using the Wizard genomic DNA purification kit (Promega, Fitchburg, WI, USA). New pairs of primers were generated for PCR amplification (Supplementary Table 1) based on the SLC6A7 gene (NM_014228) in the chromosome 5q31-32 genomic sequence (NT_029289). All exons and their flanking regions, including the promoter region $(2 \mathrm{~kb})$, were resequenced from genomic DNA from the initial 24 Korean asthmatics using the ABI PRISM 3730 DNA analyzer (Applied Biosystems, Foster City, CA, USA). Sequence variants were verified by chromatograms.

After the initial SNP survey, a total of 17 common single nucleotide polymorphisms (SNPs) with minor allele frequency (MAF) over 10\% were selected to increase the statistical power of the results. In the case of SNPs with complete LD $\left(r^{2}=1\right)$, one SNP in the regulatory region rather than an intron was selected among the SNPs. In addition to the SNPs detected from resequencing, eight additional common dbSNPs were included in the total of 17 SNPs so as to fully analyze the association between SLC6A7 and asthma. The SNPs were genotyped in 498 asthmatic patients and 303 normal controls using BeadExpress (Illumina, San Diego, CA, USA). The genotype quality score for keeping data was set at 0.25 . As a result of this process, 487 asthmatics and 286 normal controls were successfully genotyped.

\section{Statistics}

Haploview v4.1 software was used to determine LD of SNPs in SLC6A7.23 Lewontin's $D^{\prime}\left(\left|D^{\prime}\right|\right)$ and the LD coefficient $r^{2}$ between all pairs of biallelic loci were examined. ${ }^{24}$ After estimation using PHASE software, haplotypes were computed by logistic analyses using the Statistical Analysis System (SAS). Subjects with missing genotypes were excluded from the analysis of individual SNPs and haplotypes. Genotype distribution of SNPs among the asthmatics and the normal subjects was computed by logistic analyses controlling for age

Table 1 Clinical profiles of study subjects

\begin{tabular}{lcc}
\hline Clinical profiles & Asthma patients & Normal controls \\
\hline Number of subjects & 498 & 303 \\
Age (year, mean \pm s.d. (min-max)) & $49.47 \pm 15.84(11.03-87.30)$ & $47.79 \pm 15.71(5.21-83.94)$ \\
Sex (male/female) & $207 / 291$ & $122 / 181$ \\
Current smoker/ex-smoker (\%) & 34.9 & 30.1 \\
Positive rate of atopy (\%) & 62.4 & 33.1 \\
BMI (kg m ${ }^{-2}$ ) & $24.17 \pm 3.49$ & $23.87 \pm 3.01$ \\
FEV ( $_{\text {, predicted }}$ & $79.33 \pm 22.18$ & $103.09 \pm 14.77$ \\
FVC \%, predicted & $82.88 \pm 18.05$ & $93.72 \pm 12.35$ \\
Bronchodilator response (\%) & $12.03 \pm 11.73$ & $2.19 \pm 5.16$ \\
Blood eosinophils (\%) & $5.63 \pm 5.84$ & $2.39 \pm 1.84$ \\
PC2O, methacholine (mg ml-1) & $4.02 \pm 6.17$ & $24.29 \pm 2.47$ \\
Total IgE (IU ml ${ }^{-1}$ ) & $416.22 \pm 1193.16$ & $115.97 \pm 168.58$ \\
Positive rate of specific IgE (D.f., \%) & 37.55 & 16.33 \\
Positive rate of specific IgE (D.p., \%) & 46.39 & 25.17
\end{tabular}

Abbreviations: BMI, body mass index; $\mathrm{FEV}_{1}$, forced expiratory volume at 1 second; FVC, forced vital capacity.

D.f., Dermatophagoides farinae; D.p., Dermatophagoides pteronyssinus. 
(continuous value), sex (male $=0$, female $=1$ ), atopy (non-atopy $=0$, atopy $=1$ ), and smoking status (nonsmoker $=0$, ex-smoker $=1$, smoker $=2$ ) as covariates using SAS. Associations between variants and bronchodilator response were computed with regression analyses using SAS. Significant associations were indicated by a $P$-value $<0.05$. Rational selection of SNPs improves the power to detect a significant association, and it also helps determine the actual sample size needed in case-control genetic association studies. ${ }^{25,26}$ In this study, 17 common SNPs with over 10\% MAF were selected to increase statistical power for determining the association using the Power for Genetic Association Analyses (PGA) software. ${ }^{27}$ The statistical power for single associations with a falsepositive rate of $5 \%$, disease prevalence of $1 \%$, the control-to-case ratio of 0.61 and an assumed relative risk of 1.5 in a co-dominant model with an LD value of 1.0 was about $72 \%$ ( $\geqslant 80 \%$ for optimal power) for MAF $10 \%$, indicating that our samples provide the proper statistical power, but not enough to test for statistical significance of association. The effective numbers of independent marker loci in each gene were calculated to correct for multiple testing using the software SNPSpD (http://genepi.qimr.edu.au/general/daleN/SNPSpD/), which is based on the spectral decomposition $(\mathrm{SpD})$ of matrices of pairwise LD between SNPs.

\section{RESULTS}

The clinical characteristics and comparisons of asthmatic patients and normal controls are summarized in Table 1. The group of asthmatics included 207 males (41.6\%) and 291 females (58.4\%) with a mean age of $49.5 \pm 15.8$. The positive rate of atopy, blood eosinophils, total IgE and positive rate of specific IgE in asthmatics were about two to three times higher than in normal controls, whereas the PC20 methacholine level of asthmatics was lower than that of controls. Furthermore, asthmatics showed over a fivefold increase in bronchodilator response compared with controls $(P<0.05)$.

Resequencing in the initial 24 Korean asthmatic patients discovered a total of 33 genetic polymorphisms (Table 2 and Figure 1a). In addition, four novel SNPs were discovered; two in the intronic region, one synonymous SNP in an exon, and one in the $3^{\prime} \mathrm{UTR}$ region. However, these new SNPs were rare variants with frequency of less than $5 \%$, and were omitted for the next study. After the initial SNP survey, a total of 17 common SNPs with over 10\% MAF were selected, including nine SNPs that were identified by resequencing and eight additional tagging SNPs that were based on International HapMap Project (http://hapmap.ncbi.nlm.nih.gov/), to increase statistical power for association. In the case of SNPs with complete LD and over 10\% MAF, one SNP that is positioned in a regulatory region, rather than in an intron, was selected among the SNPs. These 17 SNPs were genotyped in 498 asthmatic patients and 303 normal controls (Table 2).

Successful genotypes were obtained from 487 asthmatics and 286 normal controls. Pairwise comparisons of 17 SNPs showed five tight LD blocks, and haplotypes were inferred by PHASE software (Figures $1 \mathrm{~b}$ and 2). In logistic analyses adjusted for age, sex, smoking status and atopy, all SNPs in LD block $2-5$ showed significant associations with asthma (Table $3, P<0.05$ ). In particular, the variations of $+11431 \mathrm{~T}>\mathrm{C}(\mathrm{rs} 13153971)$ in intron 6 and $+12927 \mathrm{~A}>\mathrm{G}$ (rs2240792) in intron 8 were more frequent in asthmatics than in controls showing the lowest significant association $(\mathrm{OR}=1.58 ; 95 \%$ $\mathrm{CI}=1.22-2.04$ for $+11431 \mathrm{~T}>\mathrm{C}$; $\mathrm{OR}=1.57 ; 95 \% \mathrm{CI}=1.21-2.04$ for $+12927 \mathrm{~A}>\mathrm{G}$; both $\left.P=6.0 \times 10^{-4}\right)$. For SNPs in coding and regulatory regions, the minor allele of synonymous $+13410 \mathrm{~T}>\mathrm{C}$ (Phe386Phe) was frequent in asthmatics $(P=0.01)$, whereas $+13446 \mathrm{C}>\mathrm{T}$ (Asp398Asp) was infrequent $(P=0.003)$. Also, two SNPs in the $3^{\prime} \mathrm{UTR}$ region were significantly prevalent in asthmatics $(P=0.02-$ $0.001)$. After multiple testing corrections, however, among 13 associated SNPs, only five including rs13153971, rs2240788, rs2240792, rs2240794 and rs13169575 were shown to be significantly associated (Table $3, P^{\text {corr }}<0.05$ ). In haplotype associations, haplotypes of $B L 2$, BL3-ht1, BL3-ht2, BL4-ht1, BL4-ht2 and BL5 were almost equivalent with +6343G $>A, \quad+13446 \mathrm{C}>\mathrm{T}, \quad+11431 \mathrm{~T}>\mathrm{C}, \quad+15931 \mathrm{G}>\mathrm{A}$, $+15091 \mathrm{~A}>\mathrm{G}$ and $+20113 \mathrm{~T}>\mathrm{C}$, respectively; for example, BL3-ht1 was unique to the $+13446 \mathrm{~T}$ allele among four major $B L 3$ haplotypes, and was observed to be associated with asthma.

Bronchodilator response, which is an indicator of the severity of airflow limitation in asthmatics, was found to be significantly increased in asthmatics compared with controls. Therefore, the association of variants of SLC6A7 with bronchodilator response was analyzed. In regression analyses, $+11431 \mathrm{~T}>\mathrm{C},+12213 \mathrm{C}>\mathrm{T}$ and $+12927 \mathrm{~A}>\mathrm{G}$ variants in LD block 3 and $+20113 \mathrm{~T}>\mathrm{C}$ in block 5 were associated with the bronchodilator response (Table $4, P=$ up to 0.008 for recessive model). Each heterozygous genotype brought on no increase in the bronchodilator response, whereas the homozygous genotype of each rare allele significantly increased it, indicating that the heterozygote exerts its phenotype for bronchodilator response through its recessive form, rather than its co-dominant and dominant forms. However, the significant associations of the SNPs disappeared after multiple testing corrections.

Those with atopy have a high tendency to develop asthma, as shown by about a twofold higher prevalence of atopy in asthmatics than in normal controls in this study (Table 1). In further investigating genetic effects on bronchodilator response, additional associations were observed when atopic status in asthmatics was taken into account. These additional associations included the fact that the minor alleles of variants in LD block 2 and $+13410 \mathrm{~T}>\mathrm{C}$ (Phe386Phe) in block 3 significantly increased bronchodilator response, whereas those of $+2124 \mathrm{C}>\mathrm{G}, \quad+13446 \mathrm{C}>\mathrm{T}$ (Asp398Asp), +15770T $>\mathrm{G}$ and $+15931 \mathrm{G}>\mathrm{A}$ variants decreased it (Supplementary Table 2). These associations were not found in asthmatics without atopy $(P>0.05)$.

\section{DISCUSSION}

Although genome-wide SNP screening is now available, it is still a useful strategy to investigate candidate gene(s) for disease association. Regarding this study, the chromosome region 5q31-33 contains the cytokine gene cluster, which is the most extensively studied candidate region for asthma-related traits. ${ }^{8,28,29}$ Inspired by the newly discovered link of excitatory neurotransmitters to asthma and airway remodeling for the pathogenesis of asthma, this study identified a new association between asthma and SLC6A7, which is located at chromosome 5q31-33 and encodes a neurotransmitter transporter. In addition, protocadherin 1 (PCDH1), as a mediator of cell adhesion, in chromosome 5q31-33 was identified as a novel susceptibility gene for asthma. $^{30}$ Therefore, our findings that common SNPs of the SLC6A7 gene, with tight $\mathrm{LD}$, are significantly associated with asthma and increase of the bronchodilator response suggest $S L C 6 A 7$ as a susceptibility gene for asthma.

Although the interplay between nerve and bronchial airway microvasculature is complex and not yet fully understood, it has been suggested that neurogenic inflammation might have important functions in allergic diseases such as asthma. ${ }^{31}$ Many neuropeptides have been identified to have inflammatory effects in airway diseases. ${ }^{32,33}$ An excitatory, rather than inhibitory, GABAergic system was also observed to have a key role in asthma. ${ }^{19,20}$ In addition, the GABAagonist baclofen has been reported as a potential therapeutic agent for the treatment of asthma. ${ }^{34}$ Therefore, since SLC6A7 acts as a transporter for the excitatory neurotransmitter of L-proline and has a presynaptic regulatory role in excitatory synaptic transmission, ${ }^{15,35}$ 
Table 2 Polymorphisms of SLC6A7 gene investigated in this study

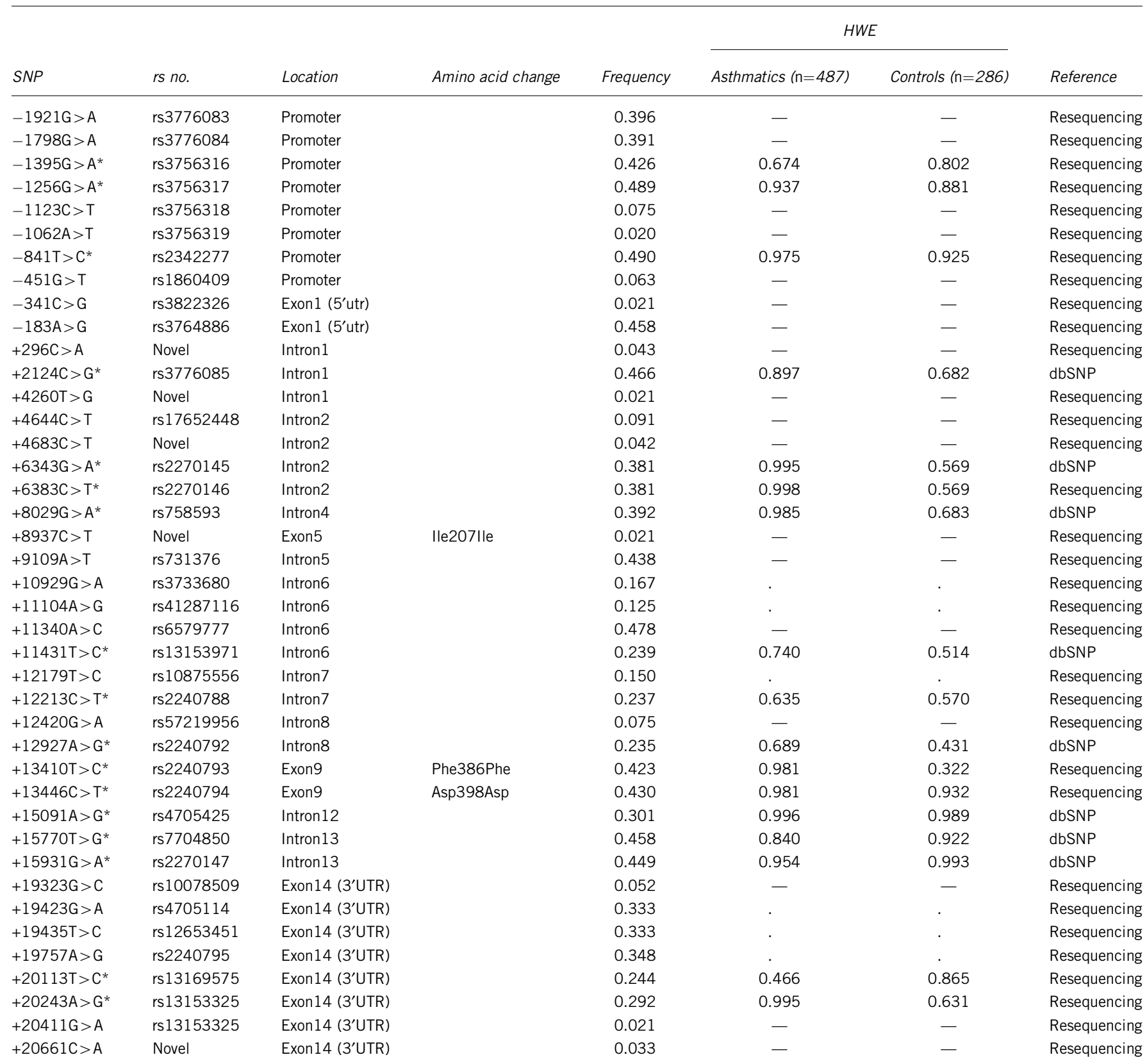

Abbreviations: HWE, Hardy-Weinberg equilibrium; UTR, untranslated region; dbSNP, public SNP of National Center for Biotechnology Information (NCBI).

Asterisks indicate SNPs that were genotyped in total case and control subjects.

The first base of the transcription start site is denoted as nucleotide +1 .

it likely has an effect on allergic and inflammatory diseases including asthma. Moreover, Pin1, a proline isomerase, was identified as an essential factor for the onset of allergic asthma, ${ }^{36}$ suggesting that L-proline-related mechanisms and variations of related genes also could contribute to asthma.

From linkage analysis of $I L-14$ and other markers in chromosome $5 q 31.1$ with total serum IgE concentration, ${ }^{37}$ genes in the chromosome $5 \mathrm{q} 31-33$ region, such as $I L-4, I L-9, I L-13$, interferon regulatory factor-1 and $\beta_{2}$-adrenergic receptor, have been reported to be associated with asthma-related phenotypes. ${ }^{9,38-40}$ More recently, genetic polymorphisms or haplotypes of the T-cell immunoglobulin and mucin domain (TIM)-1 gene were shown to confer susceptibility to asthma. ${ }^{41,42}$ The TIM-1 gene, also known as hepatitis $A$ virus cellular receptor 1 , is a recently identified gene in the chromosome $5 \mathrm{q} 31-33$ region and has been implicated in the regulation of $\mathrm{T}_{\mathrm{H}} 1-$ and $\mathrm{T}_{\mathrm{H}} 2$ cell-mediated immunity. ${ }^{43}$ Moreover, the imbalance of $\mathrm{T}_{\mathrm{H}} 1$ and $\mathrm{T}_{\mathrm{H}} 2$ immune regulation is known to affect asthma. ${ }^{44}$ However, despite these previous studies attempting to identify a link between chromosome 5q31-33 regions and asthma, no specific gene(s) thus far have been clearly implicated in the disease's pathogenesis.

Since asthmatic patients frequently have poor recognition of their symptoms, measurements of lung function provide them with a 
a Map of SLC6A7 (solute carrier family 6 (neurotransmitter transporter, L-proline), member 7) on chromosome 5q31-q32 (21.1 kb)

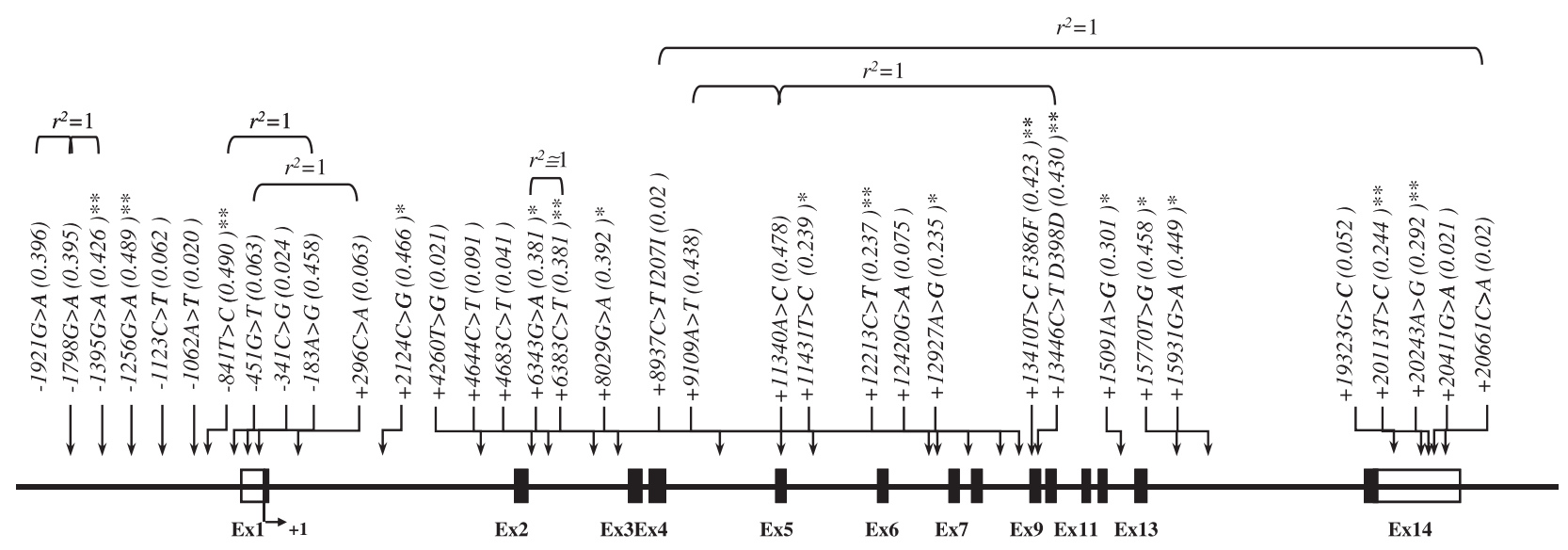

b

Haplotypes in SLC6A7

\begin{tabular}{|c|c|c|c|c|c|c|c|c|c|c|c|c|c|c|c|c|c|c|c|c|c|c|c|c|c|}
\hline \multicolumn{6}{|c|}{ Block1 } & \multicolumn{5}{|c|}{ Block2 } & \multicolumn{7}{|c|}{ Block3 } & \multicolumn{5}{|c|}{ Block4 } & \multicolumn{3}{|c|}{ Block5 } \\
\hline Hap. & 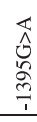 & 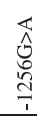 & 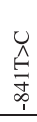 & 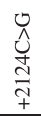 & Freq. & Hap. & 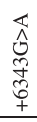 & $\begin{array}{l}\hat{\Lambda} \\
0 \\
\infty \\
0 \\
0 \\
+ \\
\end{array}$ & 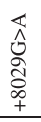 & Freq. & Hap. & 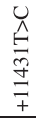 & 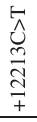 & 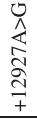 & 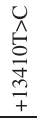 & $\begin{array}{l}\overrightarrow{\hat{A}} \\
\hat{\delta} \\
+\frac{+}{+} \\
+ \\
+\end{array}$ & Freq. & Hap. & 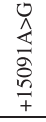 & 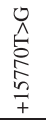 & $\begin{array}{l}\overleftrightarrow{\hat{n}} \\
\frac{\hat{\sigma}}{\sigma} \\
\frac{\sigma}{+}\end{array}$ & Freq. & Hap. & 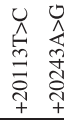 & Freq. \\
\hline ht1 & $\mathrm{G}$ & G & $\mathrm{T}$ & $\mathrm{G}$ & 0.464 & ht1 & $\mathrm{G}$ & $\mathrm{C}$ & G & 0.604 & ht1 & $\mathrm{T}$ & $\mathrm{C}$ & $\mathrm{A}$ & $\mathrm{T}$ & $\mathrm{T}$ & 0.426 & ht 1 & $\mathrm{~A}$ & $\mathrm{G}$ & A & 0.447 & ht1 & $\mathrm{T} \quad \mathrm{A}$ & 0.697 \\
\hline ht2 & $\mathrm{A}$ & A & $\mathrm{C}$ & C & 0.424 & ht2 & A & $\mathrm{T}$ & A & 0.379 & ht2 & $\mathrm{C}$ & $\mathrm{T}$ & G & C & $\mathrm{C}$ & 0.233 & ht2 & G & $\mathrm{T}$ & $\mathrm{G}$ & 0.303 & ht2 & C G & 0.230 \\
\hline ht 3 & G & A & $\mathrm{C}$ & $\mathrm{C}$ & 0.062 & ht3 & $\mathrm{G}$ & $\mathrm{C}$ & A & 0.013 & ht3 & $\mathrm{T}$ & $\mathrm{C}$ & A & $\mathrm{C}$ & $\mathrm{C}$ & 0.182 & ht3 & A & $\mathrm{T}$ & G & 0.238 & ht3 & $\mathrm{T} \quad \mathrm{G}$ & 0.060 \\
\hline ht 4 & G & G & $\mathrm{T}$ & $\mathrm{C}$ & 0.046 & ht4 & $\mathrm{G}$ & $\mathrm{T}$ & G & 0.002 & ht 4 & $\mathrm{~T}$ & C & A & $\mathrm{T}$ & $\mathrm{C}$ & 0.148 & ht 4 & A & G & G & 0.007 & ht4 & C A & 0.012 \\
\hline ht5 & $\mathrm{A}$ & A & $\mathrm{C}$ & G & 0.002 & ht5 & A & C & G & 0.001 & ht5 & $\mathrm{C}$ & $\mathrm{C}$ & A & $\mathrm{C}$ & $\mathrm{C}$ & 0.004 & ht5 & G & G & A & 0.002 & & & \\
\hline ht6 & $\mathrm{A}$ & $\mathrm{A}$ & $\mathrm{T}$ & G & 0.001 & ht6 & $\mathrm{A}$ & C & $\mathrm{A}$ & 0.001 & ht6 & $\mathrm{T}$ & $\mathrm{T}$ & A & $\mathrm{T}$ & $\mathrm{T}$ & 0.004 & ht6 & G & G & $\mathrm{G}$ & 0.002 & & & \\
\hline ht7 & $\mathrm{G}$ & A & $\mathrm{T}$ & $\mathrm{C}$ & 0.001 & & & & & & ht7 & $\mathrm{C}$ & $\mathrm{C}$ & G & $\mathrm{C}$ & $\mathrm{C}$ & 0.002 & & & & & & & & \\
\hline ht8 & $\mathrm{G}$ & $\mathrm{G}$ & $\mathrm{C}$ & $\mathrm{C}$ & 0.001 & & & & & & ht8 & $\mathrm{T}$ & $\mathrm{C}$ & G & $\mathrm{C}$ & $\mathrm{C}$ & 0.001 & & & & & & & & \\
\hline
\end{tabular}

Figure 1 Physical map and haplotypes of the SLC6A7 gene. (a) Physical map of CEP68 and its targeted SNPs. The coding exons are represented by black blocks, and 5'UTR and 3'UTR by white blocks. Numbering of SNP position +1 corresponds to the first base of the first methionine referred to NM_014228 (http://genome.ucsc.edu/). Asterisks $\left({ }^{*}\right)$ indicate the SNPs that were discovered from resequencing; ${ }^{*}$ denotes additional tagging SNPs. Complete linkage disequilibrium is denoted as $r^{2}=1$. UTR, untranslated region. (b) Haplotypes of 17 SNPs in the SLC6A7 gene. Associations of haplotypes with frequency $>0.05$ are shown in Table 3.

diagnostic confidence of asthma. Among several methods to assess airflow limitation, the response to an inhaled bronchodilator, such as the measurement within minutes after inhalation of a rapid-acting bronchodilator, is a useful indicator for the diagnosis of asthma. ${ }^{45,46}$ Although the significance disappeared after multiple testing correction, the association of variants of SLC6A7 with bronchodilator response showed a possibility that homozygous rare alleles of four SNPs, $+11431 \mathrm{~T}>\mathrm{C},+12213 \mathrm{C}>\mathrm{T},+12927 \mathrm{~A}>\mathrm{G}$ and $+20113 \mathrm{~T}>\mathrm{C}$, could increase the bronchodilator response in the recessive model. In particular, considering that the frequencies of $B L 3-h t 2$ (unique to $+11431 \mathrm{~T}>\mathrm{C},+12213 \mathrm{C}>\mathrm{T}$ and $+12927 \mathrm{~A}>\mathrm{G}$ ) and $B L 5$ (equivalent with $+20113 \mathrm{~T}>\mathrm{C}$ ) in asthmatics were higher than those of normal controls, the BL3-ht2 and BL5 haplotypes of SLC6A7 could functionally affect asthma susceptibility.

In the case of the exon region, this study found that two synonymous SNPs, +13410T $>\mathrm{C}$ (Phe386Phe) and $+13446 \mathrm{C}>\mathrm{T}$ (Asp398Asp), were frequently and infrequently associated with asthma, respectively. Recently, the tissue-specific differences in transfer RNA expression and the tissue-specific codon usage of the human gene have been investigated because codon-mediated translational control may be an important step in regulating the expression of genes. ${ }^{47,48}$ In fact, a synonymous polymorphism of the multidrug resistance 1 gene was elucidated to alter drug and inhibitor interactions. ${ }^{49}$ The amino acids Phe386Phe and Asp398Asp in the SLC6A7 protein were predicted to be positioned at transmembrane alphahelical domain 8 , and the frequencies of phenylalanine and aspartic acid were found to be different for the alpha helix region depending on synonymous codons (Supplementary Figure 1). ${ }^{50}$ Thus, different transfer RNA expression for codon usage could have an effect on the activity of the protein and the subsequent susceptibility to asthma.

The alternative splicing database creates a database of alternative splice events on genome-wide scale. ${ }^{51}$ Using an EMBL-EBI splice site prediction tool (http://www.ebi.ac.uk/asd-srv/wb.cgi?method=2), which computes positions and scores of branch point in intronic sequence for alternative splicing, the substitution from $G$ to $A$ of rs2270145, the fourth position of branch site of TTG(t/a)G, was predicted as a possible branch point adenosine with a score of 3.41 (Supplementary Figure 2). However, further experimental studies for its functions are needed to determine whether the predicted alternative splicing could have effects on asthma. Also, in additional analysis of LD near SLC6A7 in Asian populations (Japanese and Chinese) from the International HapMap Project, the SLC6A7 gene showed no LD with other genes in chromosome 5q31-33 region (Supplementary Figure 3). 


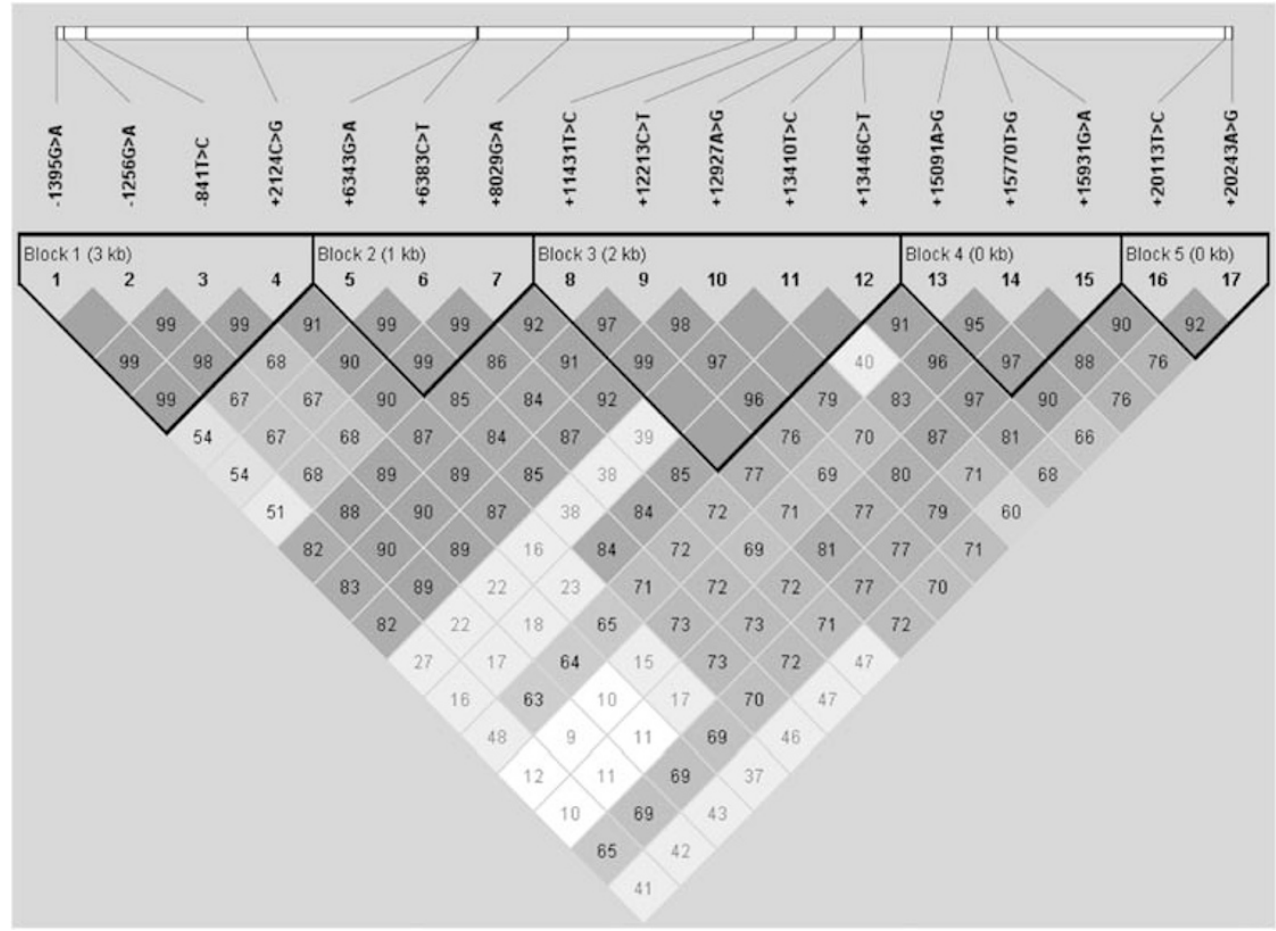

Figure 2 LD and haplotype block analysis of 17 SLC6A7 polymorphisms in a Korean population. LD plot prepared using Haploview v4.1 software downloaded from the Broad Institute (http://www.broadinstitute.org/mpg/haploview). The numbers indicate pairwise $r^{2}$ values shown as a percentage. A full color version of this figure is available at the Journal of Human Genetics journal online.

Table 3 Logistic regression analysis of SLC6A7 polymorphisms between asthmatics and controls

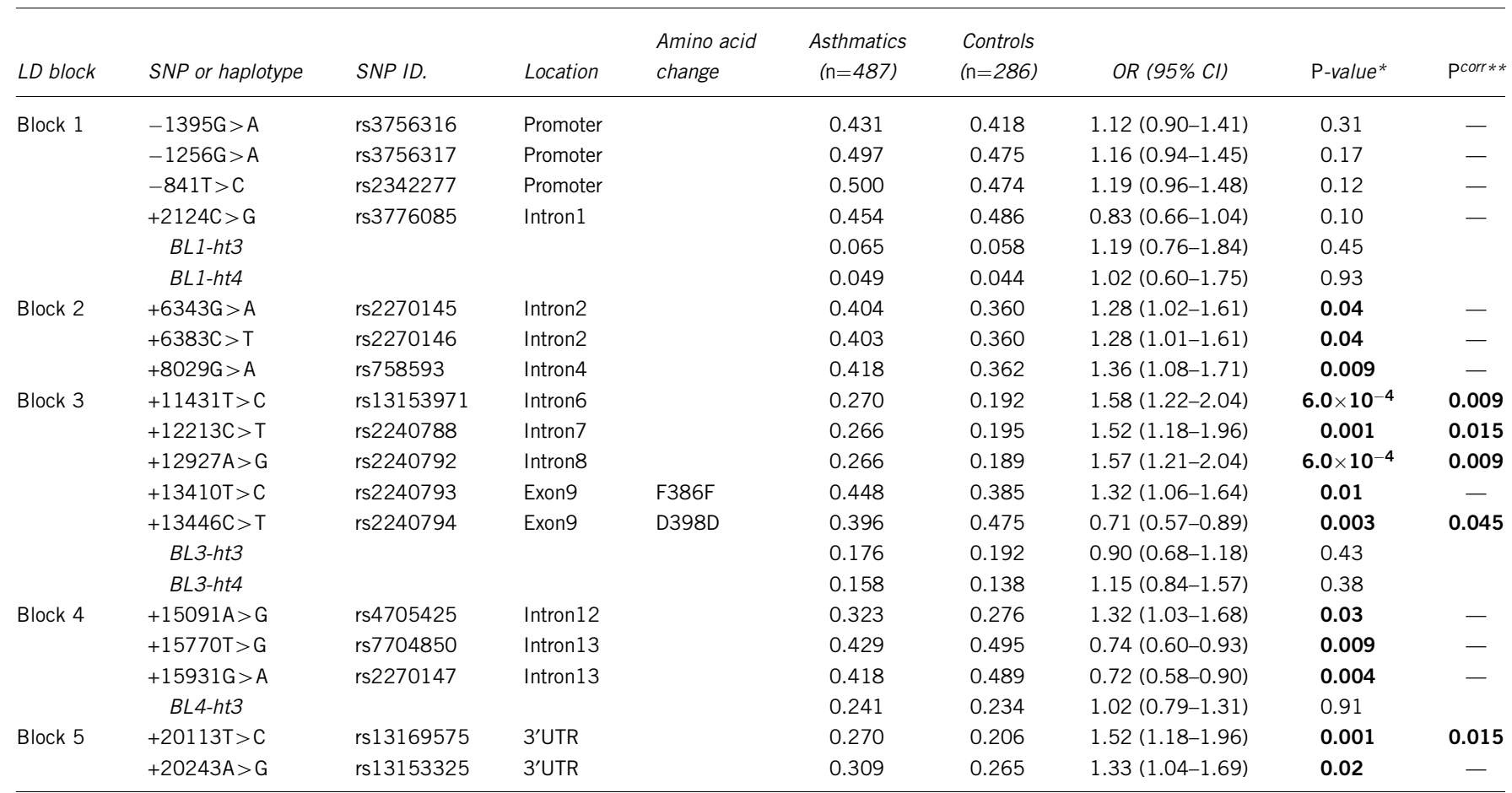

BL1-ht1, BL1-ht2, BL3-ht1, BL3-ht2, BL4-ht1 and BL4-ht2 were almost equivalent with +2124C > G, -1395G >A, 13446C > T, 11431T >C, 15931G >A and 15091A > G, respectively. Haplotypes of $B L 2$ and $B L 5$ were also equivalent with $6343 G>A$ and $20113 T>C$, respectively.

Bold values indicate the statistical significance $(P<0.05)$

${ }^{*} P$-values of the co-dominant model were obtained by logistic analysis, controlled for age, sex, smoking status and atopy as co-variables.

$* *$ porr values after multiple testing correction. 
Table 4 Regression analysis of SLC6A7 polymorphisms with bronchodilator response adjusted for age, sex, smoking and atopic status

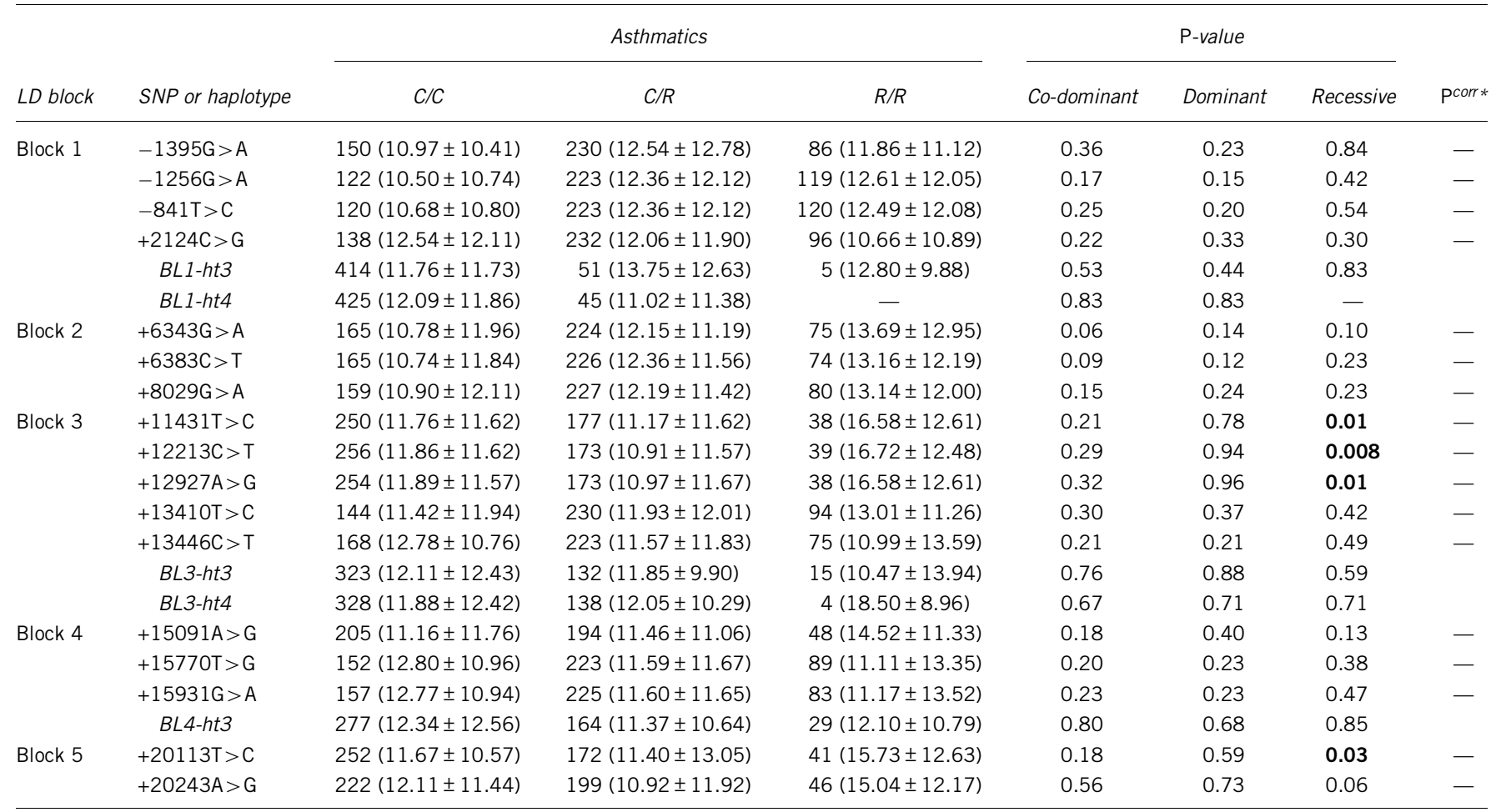

$\mathrm{C} / \mathrm{C}, \mathrm{C} / \mathrm{R}$, and R/R indicate the homozygote of the common allele, and the heterozygote and homozygote of the rare allele, respectively. Bold values indicate the statistical significance $(P<0.05)$.

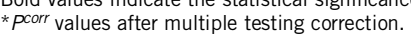

Atopy is considered to be an important risk factor in the occurrence of asthma. ${ }^{52}$ It has been reported that the prevalence of asthmatic patients is high in the populations with atopy. ${ }^{53}$ In addition, many studies have observed the association of genetic variations with asthma and atopy. ${ }^{29,54}$ In a comparison between atopic and non-atopic asthmatics, this study found that additional alleles and haplotypes were significantly associated with atopic asthma, suggesting that genetic variations of the SLC6A7 gene also could be a causative factor in the development of atopy. For this reason, additional association studies between SLC6A7 and atopy are called for.

\section{ACKNOWLEDGEMENTS}

This work was supported by a grant from the Korea Health 21 R\&D Project (A010249) and by Grant number M1-0302-00-0073 from the Korea Science and Engineering Foundation (KOSEF), funded by the Korean government (Ministry of Education, Science and Technology [MEST]) (No. 2009-0080157), and a Priority Research Centers Program through the National Research Foundation of Korea (NRF) funded by the Ministry of Education, Science and Technology (2009-0093822). The DNA samples were generously provided by the Soonchunhyang University, Bucheon Hospital Biobank, a member of the National Biobank of Korea, supported by the Ministry of Health, Welfare and Family Affairs, Republic of Korea.

1 Kay, A. B., Phipps, S. \& Robinson, D. S. A role for eosinophils in airway remodelling in asthma. Trends Immunol. 25, 477-482 (2004).

2 Sengler, C., Lau, S., Wahn, U. \& Nickel, R. Interactions between genes and environmental factors in asthma and atopy: new developments. Respir. Res. 3, 7 (2002).

3 Van Eerdewegh, P., Little, R. D., Dupuis, J., Del Mastro, R. G., Falls, K., Simon, J. et al. Association of the ADAM33 gene with asthma and bronchial hyperresponsiveness. Nature 418, 426-430 (2002).
4 Moffatt, M. F., Kabesch, M., Liang, L., Dixon, A. L., Strachan, D., Heath, S. et al. Genetic variants regulating ORMDL3 expression contribute to the risk of childhood asthma. Nature 448, 470-473 (2007).

5 Himes, B. E., Hunninghake, G. M., Baurley, J. W., Rafaels, N. M., Sleiman, P., Strachan, D. P. et al. Genome-wide association analysis identifies PDE4D as an asthma-susceptibility gene. Am. J. Hum. Genet. 84, 581-593 (2009).

6 Postma, D. S., Bleecker, E. R., Amelung, P. J., Holroyd, K. J., Xu, J., Panhuysen, C. I. et al. Genetic susceptibility to asthma-bronchial hyperresponsiveness coinherited with a major gene for atopy. N. Engl. J. Med. 333, 894-900 (1995).

7 Rioux, J. D., Daly, M. J., Silverberg, M. S., Lindblad, K., Steinhart, H., Cohen, Z. et al. Genetic variation in the 5 q31 cytokine gene cluster confers susceptibility to Crohn disease. Nat. Genet. 29, 223-228 (2001).

8 Ryu, H. J., Jung, H. Y., Park, J. S., Ryu, G. M., Heo, J. Y., Kim, J. J. et al. Gene-based single nucleotide polymorphisms and linkage disequilibrium patterns of 29 asthma candidate genes in the chromosome 5q31-33 region in Koreans. Int. Arch. Allergy Immunol. 139, 209-216 (2006).

9 Holloway, J. W., Lonjou, C., Beghe, B., Peng, Q., Gaunt, T. R., Gomes, I. et al. Linkage analysis of the 5 q31-33 candidate region for asthma in 240 UK families. Genes Immun. 2, 20-24 (2001).

10 Black, S., Teixeira, A. S., Loh, A. X., Vinall, L., Holloway, J. W., Hardy, R. et al. Contribution of functional variation in the IL13 gene to allergy, hay fever and asthma in the NSHD longitudinal 1946 birth cohort. Allergy 64, 1172-1178 (2009).

11 Early, S. B., Huyett, P., Brown-Steinke, K., Borish, L. \& Steinke, J. W. Functional analysis of -351 interleukin-9 promoter polymorphism reveals an activator controlled by NF-kappaB. Genes Immun. 10, 341-349 (2009).

12 Shimokawa, N., Nishiyama, C., Hirota, T., Tamari, M., Hara, M., Ikeda, S. et al. Functional analysis of a polymorphism in the promoter region of the IL-12/23p40 gene. Clin. Exp. Allergy 39, 228-235 (2009).

13 Kim, Y., Park, C. S., Shin, H. D., Choi, J. W., Cheong, H. S., Park, B. L. et al. A promoter nucleotide variant of the dendritic cell-specific DCNP1 associates with serum IgE levels specific for dust mite allergens among the Korean asthmatics. Genes Immun. 8, 369378 (2007).

14 Shafqat, S., Velaz-Faircloth, M., Henzi, V. A., Whitney, K. D., Yang-Feng, T. L., Seldin, M. F. et al. Human brain-specific L-proline transporter: molecular cloning, functional expression, and chromosomal localization of the gene in human and mouse genomes. Mol. Pharmacol. 48, 219-229 (1995).

15 Velaz-Faircloth, M., Guadano-Ferraz, A., Henzi, V. A. \& Fremeau, R. T. Jr. Mammalian brain-specific L-proline transporter. Neuronal localization of mRNA and enrichment of transporter protein in synaptic plasma membranes. J. Biol. Chem. 270, 15755-15761 (1995). 
16 Renick, S. E., Kleven, D. T., Chan, J., Stenius, K., Milner, T. A., Pickel, V. M. et al. The mammalian brain high-affinity L-proline transporter is enriched preferentially in synaptic vesicles in a subpopulation of excitatory nerve terminals in rat forebrain. $J$. Neurosci. 19, 21-33 (1999).

17 Warner, S. M. \& Knight, D. A. Airway modeling and remodeling in the pathogenesis of asthma. Curr. Opin. Allergy Clin. Immunol. 8, 44-48 (2008).

18 Gosens, R., Zaagsma, J., Grootte Bromhaar, M., Nelemans, A. \& Meurs, H. Acetylcholine: a novel regulator of airway smooth muscle remodelling? Eur. J. Pharmacol. 500, 193-201 (2004).

19 Xiang, Y. Y., Wang, S., Liu, M., Hirota, J. A., Li, J., Ju, W. et al. A GABAergic system in airway epithelium is essential for mucus overproduction in asthma. Nat. Med. 13, 862867 (2007).

20 Lu, W. Y. \& Inman, M. D. Gamma-aminobutyric acid nurtures allergic asthma. Clin. Exp. Allergy 39, 956-961 (2009).

21 Global Initiative for Asthma (GINA) Global strategy for asthma management and prevention. NHLBIWHO Workshop Report (National Institutes of Health, National Heart, Lung, and Blood Institute, Bethesda, MD. NIH publication, 1995).

22 Pellegrino, R., Viegi, G., Brusasco, V., Crapo, R. O., Burgos, F., Casaburi, R. et al. Interpretative strategies for lung function tests. Eur. Respir. J. 26, 948-968 (2005).

23 Barrett, J. C., Fry, B., Maller, J. \& Daly, M. J. Haploview: analysis and visualization of LD and haplotype maps. Bioinformatics 21, 263-265 (2005).

24 Hedrick, P. W. Gametic disequilibrium measures: proceed with caution. Genetics 117, 331-341 (1987).

25 Gordon, D. \& Finch, S. J. Factors affecting statistical power in the detection of genetic association. J. Clin. Invest. 115, 1408-1418 (2005).

26 Ioannidis, J. P., Trikalinos, T. A. \& Khoury, M. J. Implications of small effect sizes of individual genetic variants on the design and interpretation of genetic association studies of complex diseases. Am. J. Epidemiol. 164, 609-614 (2006).

27 Menashe, I., Rosenberg, P. S. \& Chen, B. E. PGA: power calculator for case-control genetic association analyses. BMC Genet. 9, 36 (2008).

28 Kauppi, P., Lindblad-Toh, K., Sevon, P., Toivonen, H. T., Rioux, J. D., Villapakkam, A. et al. A second-generation association study of the $5 q 31$ cytokine gene cluster and the interleukin-4 receptor in asthma. Genomics 77, 35-42 (2001).

29 Donfack, J., Schneider, D. H., Tan, Z., Kurz, T., Dubchak, I., Frazer, K. A. et al. Variation in conserved non-coding sequences on chromosome $5 q$ and susceptibility to asthma and atopy. Respir. Res. 6, 145 (2005).

30 Koppelman, G. H., Meyers, D. A., Howard, T. D., Zheng, S. L., Hawkins, G. A., Ampleford, E. J. et al. Identification of PCDH1 as a novel susceptibility gene for bronchial hyperresponsiveness. Am. J. Respir. Crit. Care Med. 180, 929-935 (2009).

31 Pisi, G., Olivieri, D. \& Chetta, A. The airway neurogenic inflammation: clinical and pharmacological implications. Inflamm. Allergy Drug Targets 8, 176-181 (2009).

32 Barnes, P. J. Neuropeptides and asthma. Am. Rev. Respir. Dis. 143, S28-S32 (1991).

33 Barnes, P. J. Neurogenic inflammation in the airways. Respir. Physiol. 125, 145-154 (2001).

34 Dicpinigaitis, P. V., Spungen, A. M., Bauman, W. A., Absgarten, A. \& Almenoff, P. L. Inhibition of bronchial hyperresponsiveness by the GABA-agonist baclofen. Chest 106, 758-761 (1994).

35 Felix, D. \& Kunzle, H. The role of proline in nervous transmission. Adv. Biochem. Psychopharmacol. 15, 165-173 (1976).

36 Shen, Z. J., Esnault, S. \& Malter, J. S. The peptidyl-prolyl isomerase Pin1 regulates the stability of granulocyte-macrophage colony-stimulating factor mRNA in activated eosinophils. Nat. Immunol. 6, 1280-1287 (2005).
37 Marsh, D. G., Neely, J. D., Breazeale, D. R., Ghosh, B., Freidhoff, L. R., EhrlichKautzky, E. et al. Linkage analysis of IL4 and other chromosome 5q31.1 markers and total serum immunoglobulin E concentrations. Science 264, 1152-1156 (1994).

38 Lipworth, B. J., Hall, I. P., Tan, S., Aziz, I. \& Coutie, W. Effects of genetic polymorphism on ex vivo and in vivo function of beta2-adrenoceptors in asthmatic patients. Chest 115, 324-328 (1999).

39 Ober, C. \& Hoffjan, S. Asthma genetics 2006: the long and winding road to gene discovery. Genes Immun. 7, 95-100 (2006).

40 Yokouchi, Y., Nukaga, Y., Shibasaki, M., Noguchi, E., Kimura, K., Ito, S. et al. Significant evidence for linkage of mite-sensitive childhood asthma to chromosome 5q31-q33 near the interleukin 12 B locus by a genome-wide search in Japanese families. Genomics 66, 152-160 (2000).

41 Wu, Q. W., Cai, P. C., Wang, L., Li, Y. R., Kong, L. L. \& Hu, L. H. Family-based association study of Tim-1 and Tim-3 gene polymorphisms with childhood asthma in Chinese trios. Int. Arch. Allergy Immunol. 150, 252-260 (2009).

$42 \mathrm{Wu}, \mathrm{Q} ., \mathrm{Hu}, \mathrm{L} ., \mathrm{Cai}, \mathrm{P} ., \mathrm{Li}, \mathrm{Y}$. , Chen, F. \& Kong, L. Association analysis of TIM-1 -232G $>$ A and 5383 5397 insertion/deletion polymorphisms with childhood asthma and total serum immunoglobulin E levels in middle China. J. Investig. Allergol. Clin. Immunol. 19, 146-153 (2009).

43 Meyers, J. H., Chakravarti, S., Schlesinger, D., Illes, Z., Waldner, H., Umetsu, S. E. et al. TIM-4 is the ligand for TIM-1, and the TIM-1-TIM-4 interaction regulates T cell proliferation. Nat. Immunol. 6, 455-464 (2005).

44 Renauld, J. C. New insights into the role of cytokines in asthma. J. Clin. Pathol. 54, 577-589 (2001).

45 Taylor, D. R. \& Kennedy, M. A. Genetic variation of the beta(2)-adrenoceptor: its functional and clinical importance in bronchial asthma. Am. J. Pharmacogenomics 1, 165-174 (2001).

46 Corvol, H., De Giacomo, A., Eng, C., Seibold, M., Ziv, E., Chapela, R. et al. Genetic ancestry modifies pharmacogenetic gene-gene interaction for asthma. Pharmacogenet. Genomics 19, 489-496 (2009).

47 Plotkin, J. B., Robins, H. \& Levine, A. J. Tissue-specific codon usage and the expression of human genes. Proc. Natl. Acad. Sci. USA 101, 12588-12591 (2004).

48 Dittmar, K. A., Goodenbour, J. M. \& Pan, T. Tissue-specific differences in human transfer RNA expression. PLoS Genet. 2, e221 (2006).

49 Kimchi-Sarfaty, C., Oh, J. M., Kim, I. W., Sauna, Z. E., Calcagno, A. M., Ambudkar, S. V. et al. A 'silent' polymorphism in the MDR1 gene changes substrate specificity. Science 315, 525-528 (2007).

50 Gupta, S. K., Majumdar, S., Bhattacharya, T. K. \& Ghosh, T. C. Studies on the relationships between the synonymous codon usage and protein secondary structural units. Biochem. Biophys. Res. Commun. 269, 692-696 (2000).

51 Stamm, S., Riethoven, J. J., Le Texier, V., Gopalakrishnan, C., Kumanduri, V., Tang, Y. et al. ASD: a bioinformatics resource on alternative splicing. Nucleic. Acids Res. 34 D46-D55 (2006).

52 Ronchetti, R., Rennerova, Z., Barreto, M. \& Villa, M. P. The prevalence of atopy in asthmatic children correlates strictly with the prevalence of atopy among nonasthmatic children. Int. Arch. Allergy Immunol. 142, 79-85 (2007).

53 Ronchetti, R., Jesenak, M., Rennerova, Z., Barreto, M., Ronchetti, F. \& Villa, M. P. Relationship between atopic asthma and the population prevalence rates for asthma or atopy in children: atopic and nonatopic asthma in epidemiology. Allergy Asthma Proc. 30, 55-63 (2009).

54 Contopoulos-loannidis, D. G., Kouri, I. N. \& Ioannidis, J. P. Genetic predisposition to asthma and atopy. Respiration 74, 8-12 (2007).

Supplementary Information accompanies the paper on Journal of Human Genetics website (http://www.nature.com/jhg) 